\title{
ADAPTACIÓN Y ESTABILIDAD DEL RENDIMIENTO EN GENOTIPOS DE FRIJOL AZUFRADO EN EL SUR DE SONORA, MÉXICO
}

\section{ADAPTATION AND YIELD STABILITY IN YELLOW GENOTYPES OF DRY BEANS AT SOUTHERN SONORA, MÉXICO}

\author{
Isidoro Padilla Valenzuela ${ }^{1^{*}}$, Rafael A. Salinas Pérez ${ }^{2}$, Jorge A. Acosta Gallegos ${ }^{3}$ y \\ Franklin G. Rodríguez Cota
}

\begin{abstract}
${ }^{1}$ Sitio Experimental Valle del Mayo, Instituto Nacional de Investigaciones Forestales, Agrícolas y Pecuarias (INIFAP). Carr. Navojoa-Huatabampo km 9. Apdo. Postal 189. 85800, Navojoa, Son. Tel. y Fax 01 (642) 423-30-45 Ext. 106. ${ }^{2}$ Campo Experimental Valle del Fuerte, INIFAP. Carr. Internacional km. 1609. Apdo. Postal 342, Juan José Ríos, Sin. ${ }^{3}$ Campo Experimental Bajío, INIFAP. Km. 6.5 Carr. Celaya-San Miguel Allende. Apdo. Postal 38010.
\end{abstract}

*Autor para correspondencia (padilla.isidoro@inifap.gob.mx)

\section{RESUMEN}

En Sonora, México se cultiva frijol (Phaseolus vulgaris L.) en 7600 ha de donde se obtienen 11600 t, y se estima una demanda estatal de $50000 \mathrm{t}$ anuales. El frijol se siembra en dos épocas: otoñoinvierno y primavera-verano en el sur de Sonora en riego, y contribuye con 70 a $80 \%$ de la superficie estatal. Las variedades predominantes son de grano grande ( $>40 \mathrm{~g} / 100$ semillas) y de color amarillo que ocupan el $90 \%$ del área. Las tres principales variedades son: 'Azufrado Peruano 87' (42 \%), 'Azufrado Pimono 78' (27\%) y 'Azufrado Higuera 94' (13\%). Entre las limitantes importantes de la producción de frijol azufrado en la región destacan el mosaico común (BCMV), el mosaico sureño (BSMV), el mosaico clorótico (BCIMV), el mosaico dorado (BGYMV) y el virus de la hoja enrollada de la calabaza (SLRMV), que pueden ocasionar la pérdida total de la producción. El propósito de esta investigación fue determinar la adaptación y estabilidad del rendimiento y variables relacionadas en cinco materiales de frijol azufrado. La investigación se condujo en el Valle del Mayo, Sonora, en suelo migajón limoso, durante los ciclos otoño-invierno 2002-2003, 2004-05, 2005-06 y 2006-07; las líneas avanzadas con hábito de determinado (Tipo I) fueron: Mo 94/95 1039 y Mo 94/95 1041, y los testigos comerciales 'Azufrado Higuera 94', 'Azufrado Noroeste 94' y 'Azufrado Peruano 87'. Se analizó el rendimiento y sus componentes y la relación de la virosis con poblaciones de mosquita blanca (Bemisia argentifolii Bellows \& Perring). Se usaron los valores de desviación estándar para estimar la consistencia de los genotipos, un modelo de estabilidad genotípica y una clasificación de variedades por estabilidad. Se encontró que la respuesta de las variedades es afectada por las bajas temperaturas y por la virosis asociada con grandes poblaciones de insectos vectores. En siembras de otoño-invierno bajo riego, el rendimiento depende del número y peso de la semilla y de los días a madurez fisiológica. El rendimiento más estable lo obtuvo la variedad 'Azufrado Higuera 94'.

Palabras clave: Phaseolus vulgaris, temperaturas bajas, enfermedades, riego.

\begin{abstract}
SUMMARY
In Sonora, México dry bean (Phaseolus vulgaris $L$.) is cultivated in 7600 ha from where $11600 \mathrm{t}$ are harvested. The annual state demand is estimated in $50000 \mathrm{t}$. Dry beans are grown under irrigation at two seasons: Autumn-Winter and Spring-Summer at southern Sonora, and account for 70 to $80 \%$ of the state total. The predominant varieties have large grain $(40 \mathrm{~g} / 100$ seeds) of yellow color which occupy $90 \%$ of the area. Main varieties are 'Azufrado Peruano 87' (42\%), 'Azufrado Pimono 78' (27\%) and 'Azufrado Higuera 94' $(13 \%)$. The main constraints of the yellow-colored bean production in the region are virus diseases, particularly common mosaic (BCMV), southern mosaic (BSMV), clorothic mosaic (BCIMV), golden mosaic (BGYMV) and the cucumber leaf roll virus (SLRMV), which may cause total losses in production. In this investigation we determined the adaptation and stability of the yield and related variables of five yellow-colored bean genotypes. The investigation was carried out in the Mayo Valley, on a sandy loam soil, during the 2002-2003, 2004-05, 2005-06 and 2006-07 Autumn-Winter seasons; the advanced lines of determinate type beans (Type I) were: Mo 94/95 1039 and Mo 94/95 1041, and the commercial checks were 'Azufrado Higuera 94', 'Azufrado Peruano 87' and 'Azufrado Noroeste 94'. The yield and yield components were analyzed regarding their relation to virus and to populations of whitefly (Bemisia argentifolii Bellows \& Perring). The values of standard deviation, the genotypic stability model and a classification of varieties for adaptability were used to consider the genotypes consistency. Results indicated that the varieties performance is affected by low temperatures and by viruses associated to great populations of vectors. For beans planted in the Autumn-Winter season, under irrigation, yield depends on the seed number and size and on the days to physiological maturity. Cultivar 'Azufrado Higuera 94' showed the most stable yield.
\end{abstract}

Index words: Phaseolus vulgaris, low temperatures, diseases, irrigation. 


\section{INTRODUCCIÓN}

El frijol (Phaseolus vulgaris L.) es un alimento benéfico porque aporta olisacáridos, taninos, antocianinas, ácido fítico, lectinas, daizeína y genisteína, compuestos que ayudan a prevenir varios tipos de cáncer y problemas cardiovasculares (Maldonado y Acosta, 2007; Com. personal). Para abastecer la demanda nacional, se producen 1.34 millones de toneladas anuales de grano (FAO, 2007) y se importan alrededor de $350000 \mathrm{t}$ de Estados Unidos (Rojo, 2002). En los últimos años el consumo de frijol ha disminuido a $12 \mathrm{~kg}$ anuales per capita, debido a la aparición de la comida rápida en un escenario en el que la mujer trabaja y tiene un tiempo reducido para la preparación alimentos (Cámara de Diputados, 2004).

En Sonora se cultiva frijol en 7600 ha de donde se obtienen $11600 \mathrm{t}$ anuales (SIAP, 2005). Se estima una demanda estatal de $50000 \mathrm{t}$ anuales, de las cuales $60 \%$ se cubre con frijol 'Pinto Americano' que se importa de las áreas frijoleras de Colorado y Nebraska, en EE. UU., generalmente a un mayor precio que el local. En el sur de Sonora se siembra en dos épocas: otoño-invierno y primavera-verano, ambas con riego. Las variedades predominantes en la región son de grano grande (> $40 \mathrm{~g} / 100$ semillas) y de color amarillo (azufrado), que ocupan $90 \%$ del área. Las principales variedades utilizadas son: 'Azufrado Peruano 87' (42 \%), 'Azufrado Pimono 78' (27 \%), 'Azufrado Higuera 94' (13\%), 'Azufrado Noroeste 94' (4 \%) y 'Azufrado 100' (4 \%) (CRSV, 2004). Las principales causas que limitan la producción de frijol azufrado en la región son las virosis, entre las que destacan el mosaico común (BCMV), el mosaico sureño (BSMV), el mosaico clorótico (BClMV), el mosaico dorado (BGYMV) y el virus de la hoja enrollada de la calabaza (SLRMV), que al incidir en forma combinada, pueden ocasionar la pérdida total de la producción (Padilla et al., 2000). El resto de la superficie se cubre con frijol 'Pinto Americano' o nacional. Otras causas que restringen la producción del frijol 'Pinto Americano' es la roya o chahuixtle (Uromyces appendiculatus var. appendiculatus) cuyas epifitias reducen el rendimiento entre 50 y $59 \%$ (Valenzuela y Armenta, 1990), la falta de variedades nacionales de ese tipo con calidad de grano y con larga vida de anaquel, pues la testa del grano de las variedades disponibles tiende a oscurecerse por oxidación después de tres meses de haber sido cosechado. Además debe considerarse la adaptación del frijol al clima y al suelo de las áreas de producción del sur de Sonora, porque influyen en su respuesta a las enfermedades.

El desarrollo, liberación y distribución de una nueva variedad es la fase final en un programa de fitomejoramiento. El fracaso en esta etapa podría significar la pérdi- da de todo el esfuerzo previo en el proceso de selección. Sin embargo, independientemente del trabajo realizado para el desarrollo de la nueva variedad, el éxito o fracaso de ésta depende primordialmente del productor, quien utiliza su criterio y experiencia para determinar su uso en condiciones comerciales (Welsh, 1990). El propósito de esta investigación fue determinar la adaptación y estabilidad del rendimiento y variables relacionadas, de dos líneas avanzadas y tres testigos comerciales de frijol azufrado bajo riego, en cuatro ciclos consecutivos de otoñoinvierno en el sur de Sonora.

\section{MATERIALES Y MÉTODOS}

La investigación se llevó a cabo en el Valle del Mayo, Sonora, en suelo migajón limoso, en Navolato, municipio de Etchojoa, Sonora $\left(27^{\circ} 00\right.$ de LN y $106^{\circ} 26^{\prime}$ y $109^{\circ}$ $30^{\prime}$ 'de LO), a $39 \mathrm{msnm}$, durante los ciclos otoño-invierno 2002-2003, 2004-05, 2005-06 y 2006-07. Se evaluaron dos líneas avanzadas de frijol azufrado de hábito determinado (Tipo I): Mo 94/95 1039 y Mo 94/95 1041, y tres variedades comerciales: 'Azufrado Higuera 94', 'Azufrado Noroeste 94' y 'Azufrado Peruano 87', desarrolladas en el programa de mejoramiento de frijol para el trópico seco en Juan José Ríos, Sinaloa, del Instituto Nacional de Investigaciones Forestales, Agrícolas y Pecuarias. En todos los ciclos se sembró en húmedo, en forma manual, durante la primera quincena de octubre. El manejo agronómico fue el sugerido para el cultivo en la región (Padilla et al., 2000). Durante el ciclo de cultivo se registraron datos de temperaturas máxima y mínima, humedades relativas máxima y mínima y precipitación, en las estaciones climatológicas automatizadas del Valle del Mayo y del Chapote, ambas ubicadas a una distancia aproximada de $3.5 \mathrm{~km}$ del sitio de prueba ${ }^{1}$. Además se registraron las poblaciones de mosquita blanca (Bemisia argentifolii Bellows \& Perring) mediante trampas amarillas pegajosas y charolas del mismo color con agua jabonosa.

Se midieron las variables: rendimiento de grano (RG), número de vainas por planta (VP), número de granos por vaina (GV), peso de 100 semillas (PCG), reacción a virosis (VR) y días a madurez fisiológica (MF). La calificación por virosis se hizo de acuerdo con una escala de 0 a 9 (CIAT, 1987) donde valores de 1 a 2 se consideran resistentes; 3 a 4 tolerantes; 5 a 6 moderadamente tolerantes; 7 a 8 moderadamente susceptibles; y 9 susceptibles. Para medir el rendimiento se cosecharon las plantas de 5 $\mathrm{m}$ de los dos surcos centrales $\left(8 \mathrm{~m}^{2}\right)$. Para determinar VP se tomaron cinco plantas al azar por parcela, y los GV se contaron en 20 vainas.

\footnotetext{
${ }^{1}$ Sistema de Información Agroclimática. Fundación Produce Sonora. www.agroson.org.mx (Febrero 15 de 2008).
} 
En el análisis estadístico se consideró el RG y sus componentes (VP y GV), en los que se utilizó el arreglo parcelas divididas donde la parcela principal fue el ciclo y la subparcela los genotipos, que consistió de 4 surcos de 6 $\mathrm{m} \times 0.80 \mathrm{~m}$ de ancho. Se usó la prueba de Tukey $(\alpha=$ $0.05)$ para la separación de medias. Se calcularon los coeficientes de correlación el rendimiento y sus componentes. Se usaron los valores de desviación estándar para estimar la consistencia de los genotipos (Binswanger y Barah, 1980), el modelo de estabilidad de Eberhart y Russell (1966) y la clasificación de variedades propuesta por Carballo y Márquez (1970).

\section{RESULTADOS Y DISCUSIÓN}

\section{Rendimiento de grano (RG) y sus componentes}

Se observaron diferencias significativas $(P \leq 0.05)$ entre variedades, entre ciclos y por efecto de la interacción. La variedad 'Azufrado Higuera 94' y la línea Mo 94/95 1039 fueron las de mayor rendimiento en los cuatro ciclos con 2.922 y $2.842 \mathrm{t} \mathrm{ha}^{-1}$, respectivamente (Cuadro 1). La var. 'Azufrado Peruano 87' registró el RG más bajo con $2.585 \mathrm{t} \mathrm{ha}^{-1}$, lo cual representa una diferencia de $13 \%$ con respecto al mejor testigo 'Azufrado Higuera 94' y de $10.4 \%$ con la mejor línea Mo 94/95 1039. En los ciclos 2002-03 y 2006-07 el RG fue de 3.364 y $3.311 \mathrm{t} \mathrm{ha}^{-1}$, mientras que en el ciclo 2004-05 apenas rindió $1.83 \mathrm{t} \mathrm{ha}^{-1}$ lo cual representa una reducción de $46 \%$. Dentro de ciclos los mejores rendimientos se encontraron con la línea Mo 94/95 1039 y la variedad 'Azufrado Noroeste 94' que rindieron 3.563 y $3.501 \mathrm{t} \mathrm{ha}^{-1}$ en 2002-03, y el peor fue en el ciclo 2004-05 en la variedad 'Azufrado Peruano 87' que apenas produjo $1.028 \mathrm{t} \mathrm{ha}^{-1}$. Aunque los cinco genotipos poseen el mismo hábito de crecimiento determinado y son de ciclo similar, sus respuestas variaron entre ciclos por diferencia en condiciones ambientales y en presión de enfermedades, lo que se reflejó en una interacción genotipo por ciclo significativa.

Los bajos rendimientos obtenidos en el ciclo 2004-05 se atribuyeron al incremento en las poblaciones de mosquita blanca Bemisia argentifolii (Figura 1), que disemi- naron los virus BGMV, BCIMV y SLRMV que causaron aborto de flores y vainas (Padilla et al., 2000; Schwartz y Gálvez, 1979). En cuanto a la respuesta de genotipos a la virosis (Figura 2), los de menor presencia de virosis a través de los ciclos fueron la var. 'Azufrado Higuera 94' y la línea Mo 94/95 1039, aunque en el ciclo 2004-05, que fue el de mayor incidencia, las var. 'Azufrado Noroeste 94', 'Azufrado Peruano 87' y la línea Mo 94/95 1039 mostraron altos valores de virosis (7.7). La var. 'Azufrado Peruano 87 ' resultó la más susceptible con un rendimiento $61.9 \%$ menor al de 'Azufrado Higuera 94' que fue el mejor genotipo. Es de destacar que aunque en los ciclos 2005-06 y 2006-07, la línea Mo 94/95 1041 y la var. 'Azufrado Noroeste 94' mostraron altos niveles de virosis, ello no repercutió en su producción de grano, posiblemente debido al descenso de temperatura en la etapa de floración y formación de vainas que ocasionaron remisión de síntomas. Se destaca también que en el ciclo 2006-07 los mayores rendimientos de grano correspondieron a las líneas Mo 94/95 1039 y Mo 94/95 1041 (Cuadro $1)$.

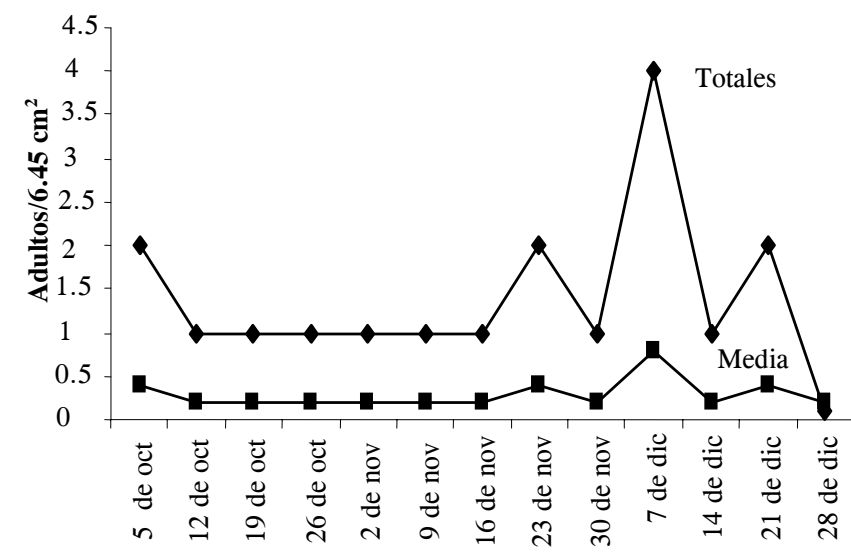

Fecha de muestreo

Figura 1. Dinámica poblacional de mosquita blanca en frijol azufrado mediante trampas amarillas. Valle de Mayo, Son. Ciclo otoñoinvierno 2004-05.

Cuadro 1. Promedio de rendimiento (t $\left.\mathrm{ha}^{-1}\right)$ de cinco genotipos de frijol azufrado a través de cuatro ciclos en el Valle del Mayo, Son. 2007.

\begin{tabular}{|c|c|c|c|c|c|}
\hline Genotipo & 2002-03 & 2004-05 & $2005-06$ & 2006-07 & Promedio \\
\hline Mo 94/95 1039 & $3.56 \mathrm{a}^{+}$ & $1.97 \mathrm{~b}$ & $2.43 \mathrm{a}$ & $3.40 \mathrm{a}$ & $2.84 \mathrm{ab}$ \\
\hline Мo 94/95 1041 & $3.07 \mathrm{c}$ & $1.72 \mathrm{c}$ & $2.59 \mathrm{a}$ & $3.40 \mathrm{a}$ & $2.70 \mathrm{bc}$ \\
\hline 'Azufrado Higuera 94' & $3.32 \mathrm{a}$ & $2.69 \mathrm{a}$ & $2.44 \mathrm{a}$ & $3.24 \mathrm{a}$ & $2.92 \mathrm{a}$ \\
\hline 'Azufrado Peruano 87' & $3.37 \mathrm{a}$ & $1.03 \mathrm{~d}$ & $2.63 \mathrm{a}$ & $3.31 \mathrm{a}$ & $2.58 \mathrm{c}$ \\
\hline Promedio & $3.36 \mathrm{a}^{++}$ & $1.83 \mathrm{c}$ & $2.53 \mathrm{~b}$ & $3.31 \mathrm{a}$ & \\
\hline
\end{tabular}

DHS entre vars $(0.05)=0.118$

DHS entre ciclos $(0.05)=0.1418$

DHS dentro de ciclos $(0.05)=0.235$

+ Medias con la misma letra en el sentido de columnas son iguales $(\mathrm{P} \leq 0.01)$

++ Medias con la misma letra en el sentido de hileras son iguales $(\mathrm{P} \leq 0.01)$ $\mathrm{CV}=13.4 \%$ 
Durante el ciclo 2005-06 todos los genotipos tuvieron un rendimiento de $2.5 \mathrm{t} \mathrm{ha}^{-1}$, tal vez porque en este ciclo ocurrieron temperaturas $2.7 \mathrm{a}-0.7{ }^{\circ} \mathrm{C}$ durante los días 17 y 18 de enero de 2006 (Figura 3), a los 96 d del cultivo, que afectaron el follaje del tercio superior e interrumpieron el desarrollo normal del grano. Una helada puede generar congelamiento letal para las plantas y la magnitud de su efecto depende de la duración de la misma y de la sensibilidad de la especie. Existen especies como el pepino (Cucumis sativus L.), cuyo umbral de daño por baja temperatura, el umbral es apenas de $+3{ }^{\circ} \mathrm{C}$; los cereales pueden soportar temperaturas de subcongelamiento; en frijol la temperatura mínima letal es de $0{ }^{\circ} \mathrm{C}$ (AFRD, 2006). El día 17 las temperaturas estuvieron por un corto periodo por abajo de $1{ }^{\circ} \mathrm{C}$, y el día 18 permanecieron así alrededor de $2.5 \mathrm{~h}$ con el consecuente daño a las plantas y la disminución del rendimiento. Según Bliss (1980), en frijol las temperaturas óptimas deben ser entre 15 a $27^{\circ}$ $\mathrm{C}$ durante el día y de 10 a $21^{\circ} \mathrm{C}$ durante la noche. La alta humedad relativa alta favorece un mayor cuajado de frutos, pero también la diseminación de enfermedades foliares. En el presente estudio se observó que las mejores condiciones para el desarrollo reproductivo del frijol ocurrieron en los ciclos 2002-03 y en 2006-07 (Figuras 4 y
5), en los que las temperaturas mínimas fluctuaron de 5 a $15^{\circ} \mathrm{C}$ y la máximas de 25 a $32{ }^{\circ} \mathrm{C}$, y la humedad relativa máxima se mantuvo por arriba de $90 \%$. La lluvia y la presencia de nubosidad fueron dos factores determinantes en la prevención de daños por baja temperatura y ocurrieron en la fase final del cultivo a razón de 15.7 y 24.7 mm para 2002-03 y 2006-07.

En el número de vainas por planta (VP), no hubo diferencia entre genotipos en promedio de ciclos, pero sí entre ciclos y dentro de ellos (Cuadro 2). El ciclo en el que se produjo la mayor cantidad de vainas fue el 2005-06, con una media de 23.8, seguido del ciclo 2006-07 con 18.3 vainas, ambos con diferencia significativa; los ciclos 2002-03 y 2004-05 fueron inferiores, con 15.7 y 15.3 vainas, respectivamente. Dentro de ciclos las mayores diferencias ocurrieron en 2004-05 donde sobresalieron 'Azufrado Noroeste 94', 'Azufrado Peruano 87' y la línea Mo 94/95 1041, con 18.2, 15.2 y $16.6 \mathrm{VP}$, respectivamente. Desafortunadamente, las bajas temperaturas que se registraron durante el ciclo 2005-06 interrumpieron el llenado del grano, porque afectaron entre 20 y $30 \%$ del follaje del tercio superior del dosel (datos no mostrados) y, en consecuencia, el rendimiento.

Cuadro 2. Promedio de vainas por planta en cinco genotipos de frijol azufrado a través de cuatro ciclos en el Valle del Mayo, Son. 2007.

\begin{tabular}{|c|c|c|c|c|c|}
\hline Genotipo & 2002-03 & 2004-05 & 2005-06 & 2006-07 & Media de vars. \\
\hline Mo 94/95 1039 & $17.3 \mathrm{a}^{+}$ & $12.6 \mathrm{~b}$ & $22.8 \mathrm{~b}$ & $19.2 \mathrm{a}$ & $17.9 \mathrm{a}$ \\
\hline Мо 94/95 1041 & $14.9 \mathrm{a}$ & $16.6 \mathrm{ab}$ & $22.8 \mathrm{~b}$ & $16.5 \mathrm{a}$ & $17.7 \mathrm{a}$ \\
\hline ‘Azufrado Higuera 94’ & $14.6 \mathrm{a}$ & $13.8 \mathrm{~b}$ & $27.1 \mathrm{a}$ & $18.9 \mathrm{a}$ & $18.6 \mathrm{a}$ \\
\hline ‘Azufrado Peruano 87’ & $16.3 \mathrm{a}$ & $15.2 \mathrm{ab}$ & $23.5 \mathrm{~b}$ & $18.0 \mathrm{a}$ & $18.2 \mathrm{a}$ \\
\hline Promedio & $15.7 \mathrm{c}^{++}$ & $15.3 \mathrm{c}$ & $23.8 \mathrm{a}$ & $18.3 \mathrm{~b}$ & \\
\hline \multicolumn{2}{|c|}{ DHS entre vars $(0.05)=1.58$} & + Medias c & a en el se & as son igu & \\
\hline
\end{tabular}

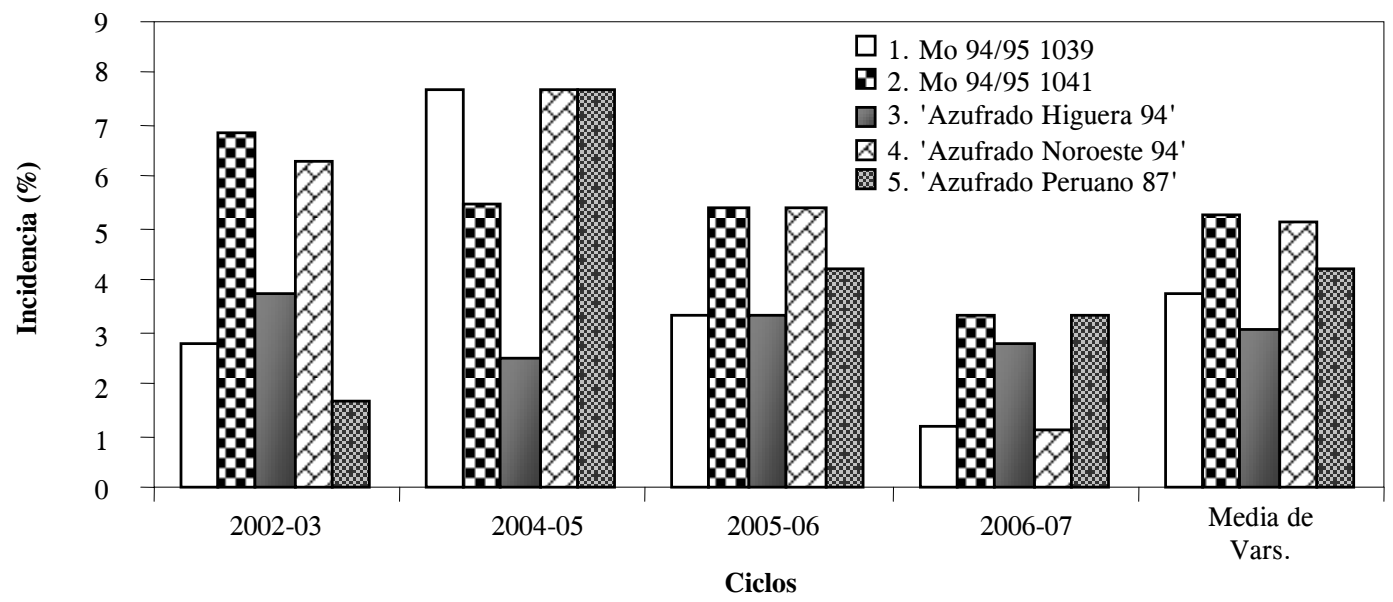

Figura 2. Incidencia de virosis en cinco genotipos de frijol azufrado a través de ciclos en el Valle del Mayo, Sonora. 2007. 


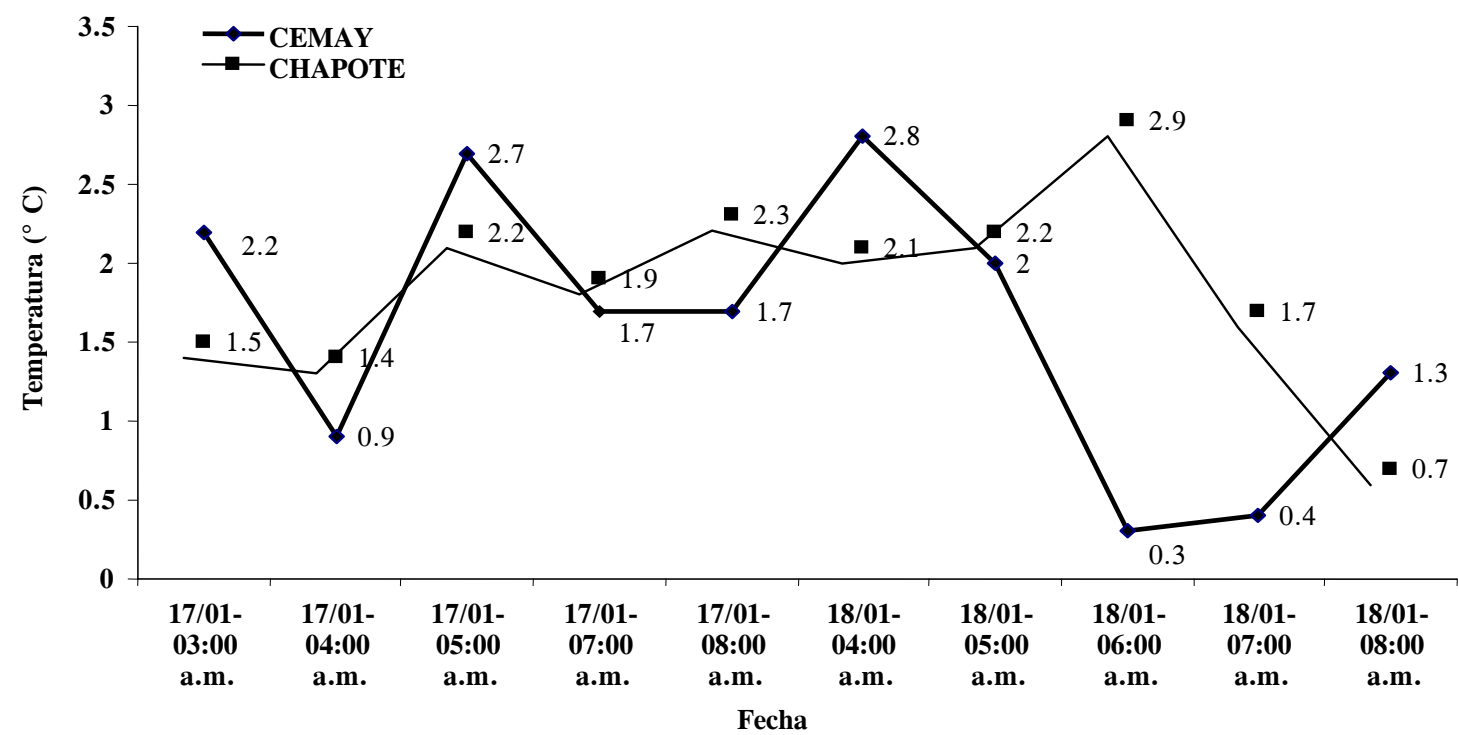

Figura 3. Temperaturas mínimas registradas en dos estaciones climatológicas automatizadas (Cemay-Chapote) los días 17 y 18 de enero de 2006. Valle del Mayo, Sonora. Ciclo otoño-invierno 2005-06.

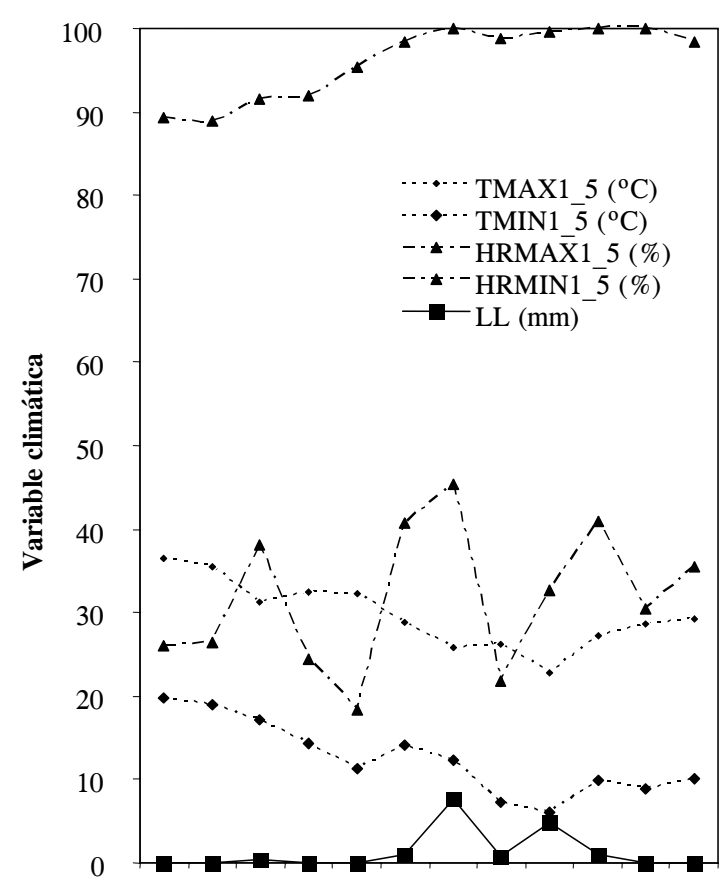

[Oct] 2002 [Nov] 2002 [Dic] 2002 [Ene] 2003

Intervalos (10 d)

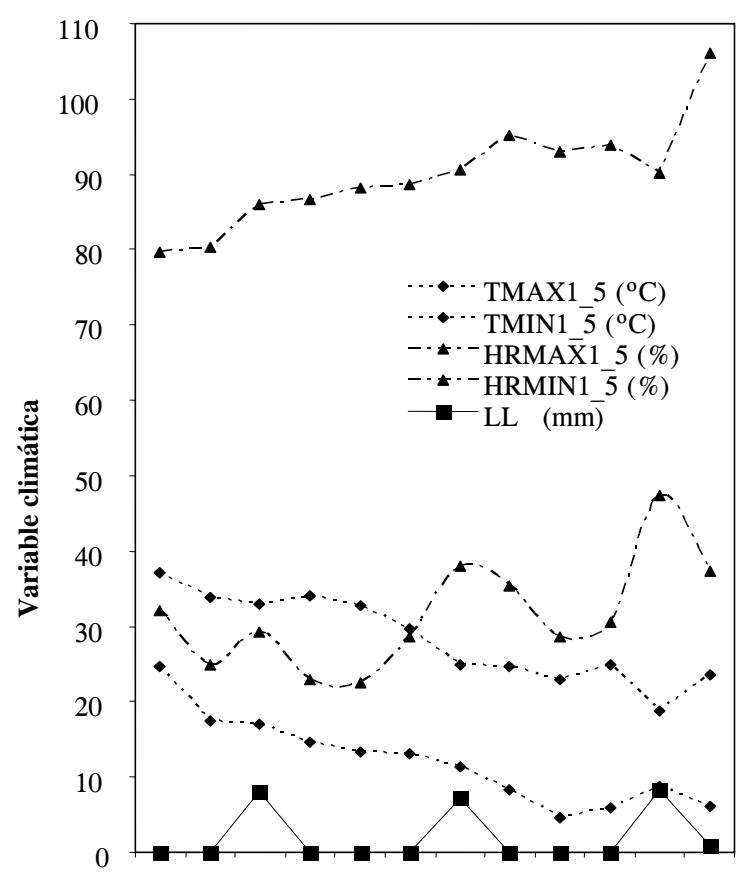

[Oct] 2006 [Nov] 2006 [Dic] 2006 [Ene] 2007

Intervalos (10 d)
Figura 4. Temperatura, humedad relativa y lluvia durante el ciclo O-I 2002-03. Valle del Mayo, Sonora.
Figura 5. Temperatura, humedad relativa y lluvia durante el ciclo O-I 2006-07. Valle del Mayo, Sonora. 
A diferencia de las vainas por planta, en el número de granos por vaina (GV) sí hubo diferencias significativas entre genotipos a través de los ciclos, donde destacaron la línea Mo 94/95 1041 y la var. 'Azufrado Peruano 87' con 4.31 y 3.96 granos (Cuadro 3). Los ciclos 2002-03 y 2005-06 fueron mejores con 4.22 granos, en comparación con el ciclo 2004-05 en el que el número de GV fue 3.64. Dentro de ciclos hubo diferencias estadísticas, excepto en 2005-06. Tanto en el ciclo 2002-03 como en el 2005-06 se obtuvieron valores de GV por arriba de cuatro en todos los genotipos.

Los datos de peso de 100 granos (PCG) arrojaron diferencias a través de ciclos, entre ciclos y dentro de éstos (Cuadro 4). El mayor tamaño de semilla se encontró en la var. 'Azufrado Higuera 94' con 45.4 g que superó a las demás; le siguieron la var. 'Azufrado Noroeste 94 ' y la línea Mo 94/95 1039 con 42.2 y 42.1 g, respectivamente. El genotipo con menor tamaño de grano $(\mathrm{P} \leq 0.05)$ fue la var. 'Azufrado Peruano 87'. Al igual que en el número de vainas por planta, las principales causas de la disminución del peso del grano fueron la helada en 2005-06 y el daño de virus en 2004-05.

\section{Madurez fisiológica (MF)}

En MF se detectaron diferencias estadísticas entre variedades, entre ciclos, dentro de éstos y en su interacción. Los genotipos más precoces fueron la var. 'Azufrado Higuera 94' y la línea Mo 94/95 1041 con 105 y 106 d, respectivamente (Cuadro 5). Los más tardíos fueron la var. 'Azufrado Peruano 87' y la línea Mo 94/95 1039 con 113 d a MF. El ciclo 2006-07 fue el más largo, y en orden descendente le siguieron 2005-06, 2002-03 y 200405, con 111, 110 y 107 d a MF, respectivamente. Dentro de ciclos, en 2004-05 la var. 'Azufrado Higuera 94' y la línea Mo 94/95 1041 fueron las más precoces con 100 y 103 d, respectivamente. El ciclo más largo ocurrió en la var. 'Azufrado Peruano 87' durante 2006-07.

\section{Asociación de variables}

El rendimiento mostró una correlación positiva y significativa $(\mathrm{P} \leq 0.05)$ con el número de granos por vaina $(\mathrm{r}$ $=0.35)$, peso de 100 gramos, PCG $(r=0.64)$ y con días a madurez fisiológica MF $(\mathrm{r}=0.22)$ (Cuadro 6). Un efecto inverso se observó con la reacción a virus VR $(\mathrm{r}=$ -0.60). El número de vainas por planta correlacionó positivamente con granos por vaina $(r=0.34)$, pero en forma negativa con respecto de PCG $(r=-0.43)$. También se detectó una correlación negativa $(r=-0.30)$ entre el PCG y VR. En un trabajo de fechas de siembra hecho en otoño-inverno en el Valle del Mayo, Sonora (Valenzuela, 1985), sólo se encontró correlación entre el rendimiento y los días a madurez, tal vez porque involucró variedades con distinto tipo y tamaño de grano. En el presente trabajo se constató dicha correlación y además, se evidenció que el rendimiento también dependió de las magnitudes de las vainas por planta, peso de 100 granos y días a madurez.

Cuadro 3. Promedio de número de granos por vaina en cinco genotipos de frijol azufrado a través de cuatro ciclos en el Valle del Mayo. 2007.

\begin{tabular}{|c|c|c|c|c|c|}
\hline Genotipo & $2002-03$ & $2004-05$ & 2005-06 & 2006-07 & Media de vars. \\
\hline Mo $94 / 951039$ & $4.0 \mathrm{~b}^{+}$ & $3.6 \mathrm{~b}$ & $4.1 \mathrm{a}$ & $3.7 \mathrm{a}$ & $3.8 \mathrm{~b}$ \\
\hline Mo $94 / 951041$ & $4.6 \mathrm{a}$ & $4.1 \mathrm{a}$ & $4.4 \mathrm{a}$ & $4.1 \mathrm{a}$ & $4.3 \mathrm{a}$ \\
\hline ‘Azufrado Higuera 94’ & $4.2 \mathrm{a}$ & $3.5 \mathrm{~b}$ & $4.4 \mathrm{a}$ & $3.5 \mathrm{~b}$ & $3.9 \mathrm{~b}$ \\
\hline 'Azufrado Peruano 87’ & $4.3 \mathrm{a}$ & $3.3 \mathrm{~b}$ & $4.2 \mathrm{a}$ & $4.1 \mathrm{a}$ & $3.9 \mathrm{~b}$ \\
\hline Promedio & $4.2 \mathrm{a}^{++}$ & $3.6 \mathrm{c}$ & $4.2 \mathrm{a}$ & $3.9 \mathrm{~b}$ & \\
\hline DHS entre vars $(0.05)=0.288$ & & misma let & tido de col & guales $(\mathrm{P}$ & \\
\hline
\end{tabular}

Cuadro 4. Promedio de peso de 100 granos (g) en cinco genotipos de frijol azufrado a través de cuatro ciclos en el Valle del Mayo. 2007.

\begin{tabular}{|c|c|c|c|c|c|}
\hline Genotipo & $2002-03$ & 2004-05 & 2005-06 & 2006-07 & Media de Vars. \\
\hline Mo 94/95 1041 & $43.0 \mathrm{~b}$ & $39.2 \mathrm{~b}$ & $35.2 \mathrm{~b}$ & $45.0 \mathrm{~b}$ & $40.6 \mathrm{c}$ \\
\hline 'Azufrado Noroeste 94’ & $45.7 \mathrm{~b}$ & $40.0 \mathrm{~b}$ & $39.0 \mathrm{a}$ & $44.0 \mathrm{~b}$ & $42.2 \mathrm{~b}$ \\
\hline ‘Azufrado Peruano 87’ & $45.2 \mathrm{~b}$ & $36.0 \mathrm{c}$ & $33.7 \mathrm{~b}$ & $43.0 \mathrm{~b}$ & $39.5 \mathrm{~d}$ \\
\hline Promedio & $46.2 \mathrm{a}^{++}$ & $39.9 \mathrm{~b}$ & $36.1 \mathrm{c}$ & $45.6 \mathrm{a}$ & \\
\hline DHS entre vars $(0.05)=1.002$ & & la mism & sentido & $\mathrm{n}$ iguales & \\
\hline
\end{tabular}


Cuadro 5. Promedio de madurez fisiológica (d) en cinco genotipos de frijol azufrado a través de cuatro ciclos en el Valle del Mayo, Son. 2007.

\begin{tabular}{|c|c|c|c|c|c|}
\hline Genotipo & $2002-03$ & 2004-05 & 2005-06 & 2006-07 & Media de Vars. \\
\hline Mo 94/95 1039 & $116 \mathrm{a}^{+}$ & $111 \mathrm{a}$ & $114 \mathrm{a}$ & $112 \mathrm{~b}$ & $113 \mathrm{a}$ \\
\hline Mo 94/95 1041 & $107 \mathrm{~b}$ & $103 \mathrm{~b}$ & $105 \mathrm{~b}$ & $107 \mathrm{c}$ & $106 \mathrm{c}$ \\
\hline 'Azufrado Noroeste 94' & $107 \mathrm{~b}$ & $110 \mathrm{a}$ & $114 \mathrm{a}$ & $114 \mathrm{~b}$ & $111 \mathrm{~b}$ \\
\hline ‘Azufrado Peruano 87’ & $109 \mathrm{~b}$ & $110 \mathrm{a}$ & $114 \mathrm{a}$ & $118 \mathrm{a}$ & $113 a b$ \\
\hline Promedio & $110 \mathrm{c}^{++}$ & $107 \mathrm{~d}$ & $111 \mathrm{~b}$ & $113 \mathrm{a}$ & \\
\hline
\end{tabular}

Cuadro 6. Matríz de correlaciones (r) entre el rendimiento, sus componentes y daño de virus en cinco genotipos de frijol azufrado a través de cuatro ciclos en el Valle del Mayo, Son. 2007.

\begin{tabular}{|c|c|c|c|c|c|}
\hline Variable & 2 & 3 & 4 & 5 & 6 \\
\hline 1. Rendimiento & $-0.035 \mathrm{~ns}$ & $0.347 * *$ & $0.643 * *$ & $0.222 *$ & $-0.601 * *$ \\
\hline 3. Granos por vaina & & & $-0.047 \mathrm{~ns}$ & $0.0009 \mathrm{~ns}$ & $-0.069 \mathrm{~ns}$ \\
\hline 5. Madurez fisiológica & & & & & -0.180 \\
\hline
\end{tabular}

6. Reacción a virosis

* Significativo $(\mathrm{P}<0.05) ; * *$ Significativo $(\mathrm{P}<0.01) ; \mathrm{ns}=$ No significativo

\section{Estabilidad de los genotipos}

Con la desviación estándar (DE) como una medida de consistencia de los genotipos a través de años o fechas de siembra (Binswanger y Barah, 1980), puede señalarse que 'Azufrado Higuera 94' cuya DE fue de 0.41 t ha $^{-1}$ (Figura 6), mostró la mayor estabilidad a través de años en el Valle del Mayo. Lo anterior puede constatarse con su comportamiento en cada uno de los ciclos y con su reacción a virosis, además, el tamaño de grano le da una ventaja comparativa con respecto al resto de los materiales. En contraste, la variedad 'Azufrado Peruano 87' con 0.99 t $\mathrm{ha}^{-1}$ de DE fue la menos estable. Desde el punto de vista lógico y convencional, un comportamiento estable es aquel que no cambia a través del tiempo y de espacio (Márquez, 1974). Sin embargo, es necesario examinar la interacción genético-ambiental con los métodos de Eberhart y Russell (1966) y de Finlay y Wilkinson (1963), que son más precisos y confiables, antes de proceder a recomendar la siembra extensiva de nuevas variedades. Estos autores determinaron un modelo que permite medir la estabilidad de cada variedad mediante la partición de la interacción genético-ambiental en dos partes: la variación debida a la respuesta de la variedad al cambiar los índices ambientales, y las desviaciones inexplicables de la regresión sobre el índice ambiental. La variedad estable es aquélla que muestra un coeficiente de regresión $b i=1 \mathrm{y}$ una desviación de regresión $S^{2} d=0$; si esta variedad presenta una media de rendimiento alta, se puede decir que es una variedad deseable. El índice ambiental se calcula para cada ambiente con el promedio de todas las variedades en un ambiente, menos la media general en todos los ambientes.

Con el criterio arriba descrito y la propuesta de clasificación propuesta por Carballo (1970) se obtuvieron los resultados que se muestran en el Cuadro 7. Así, la línea Mo 94/95 1039 y la var. 'Azufrado Noroeste 94' dan buena respuesta en todos los ambientes pero son inconsistentes, mientras que la línea Mo 94/95 1041 y la var. 'Azufrado Higuera 94' dan una mejor respuesta en ambientes desfavorables. Voysest (1985) señala que mientras que el fitomejorador se interesa en seleccionar materiales que se comportan bien en un ambiente determinado y que exhiban menos fluctuaciones cuando el ambiente cambie, el agricultor está interesado en la variedad que le rinda más en su propio ambiente. De acuerdo con Valenzuela (1985), las variedades de grano tipo peruano como 'Azufrado Peruano 87' con $b i>1.0$ aprovechan mejor los ambientes favorables, sobre todo en siembras tardías. En cambio, 'Azufrado 100' con bi $<1.0$ responde mejor en ambientes desfavorables; esta variedad se ha recomendado para siembras de temporal o secano en el sur de Sinaloa (Lépiz y Navarro, 1983). 


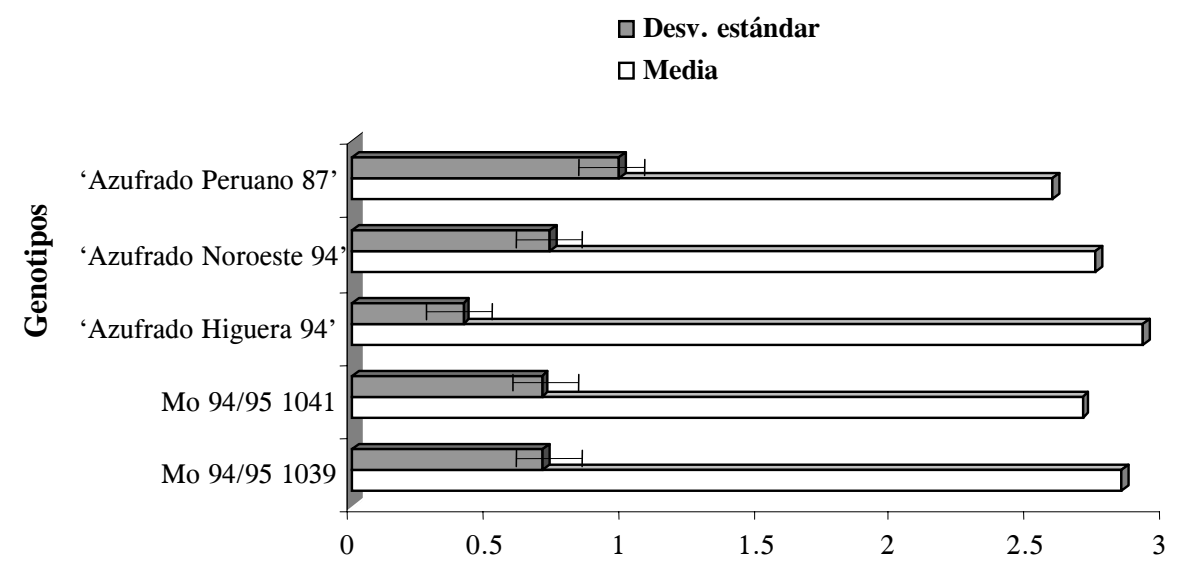

Figura 6. Rendimiento promedio y desviación estándar en frijol azufrado en el Valle del Mayo, Sonora. 2007

Cuadro 7. Clasificación de cinco genotipos de frijol en función del coeficiente de regresión $(b i)$ y desviación de regresión $\left(S^{2} d\right)$. 2007.

\begin{tabular}{lllll} 
Genotipo & Ecuación de regresión & $b i$ & $S^{2} d$ & Clasificación $\dagger$ \\
\hline Mo 94/95 1039 & $\mathrm{y}=1.0388 \mathrm{x}+2.8420$ & 1.0388 & 0.497 & $\mathrm{a}$ \\
Mo 94/95 1041 & $\mathrm{y}=0.6376 \mathrm{x}+2.4571$ & 0.6376 & 0.489 & $\mathrm{~b}$ \\
'Azufrado Higuera 94' & $\mathrm{y}=0.4642 \mathrm{x}+2.9223$ & 0.4642 & 0.168 & $\mathrm{~b}$ \\
'Azufrado Noroeste 94' & $\mathrm{y}=1.0696 \mathrm{x}+2.7434$ & 1.0696 & 0.537 & $\mathrm{a}$ \\
'Azufrado Peruano 87' & $\mathrm{y}=1.4549 \mathrm{x}+2.5850$ & 1.4549 & 0.972 & $\mathrm{c}$ \\
\hline
\end{tabular}

$\dagger: \mathrm{a}=$ Buena respuesta en todos los ambientes pero inconsistente; $\mathrm{b}=$ Mejor respuesta en ambientes desfavorables pero inconsistente; $\mathrm{c}=$ Respuesta mejor en buenos ambientes e inconsistente.

\section{CONCLUSIONES}

En la región sur de Sonora la respuesta de las variedades a través de los ciclos se vio afectada por la ocurrencia de temperaturas bajas y la virosis asociada con grandes poblaciones de Bemisia argentifolii. En siembras de otoño-invierno bajo riego, el rendimiento de grano de genotipos de frijol de hábito determinado depende principalmente del número y peso de la semilla, y de los días a madurez fisiológica. El rendimiento más estable a través de ciclos lo obtuvo la variedad 'Azufrado Higuera 94'.

\section{BIBLIOGRAFÍA}

AFRD (2006) Protection against frost damage. Agriculture, Food and Rural Development. Alberta, Canada Government. www.agric.gov.ab.ca ( $1^{\circ}$ de septiembre de 2006).

Binswanger H P, B C Barah (1980) Yield risk, risk aversion and genotype selection: Conceptual issues and approaches. ICRISAT. Res. Bull. No 3. 25 p.

Bliss F A (1980) Common Bean. In: Hybridization of Crop Plants. W R Fehr, H Hadley (eds). American Society of Agronomy. Madison, Wisconsin, USA. pp:273-284.

Cámara de Diputados (2004) Impacto de las importaciones de maíz blanco y de frijol originarias de EUA en el mercado interno de México. Informe del Centro de Estudios de las Finanzas Públicas. Palacio legislativo de San Lázaro. Noviembre de 2004. www.croplifelatinoamerica.org/ data/media/mexico (24 de Junio de 2006).
Carballo C A, F Márquez S (1970) Comparación de variedades de maíz del Bajío y de la mesa central por su rendimiento y estabilidad. Agrociencia 5:129-146.

CIAT (1979) Problemas de Producción de Frijol: Enfermedades, Limitaciones Edáficas y Climáticas de Phaseolus vulgaris. F H Schwartz, G E Gálvez (eds). Cali, Colombia. pp:211-298.

Centro Internacional de Agricultura Tropical, CIAT (1987) Sistema estándar para la evaluación de germoplasma de frijol. A Schoonchoven, M A Pastor C (comps). Cali, Colombia. $56 \mathrm{p}$.

Cuéllar E I, F Ibarra J, J Muñoz J, M Valdéz J, A Castillo, A Pajarito (1998) Tecnologías para Aumentar la Productividad de Frijol en Durango. Publicación Especial No 12. Campo Experimental Valle del Guadiana. CIRNE-INIFAP. 54 p.

CSRV (2004) Datos Estadísticos del Comité Regional de Sanidad Vegetal. Navojoa, Sonora, México. (www. cesaveson.com).

Eberhart S A, W A Russell (1966) Stability parameters for comparing varieties. Crop Sci. 6:36-40.

FAO (2007) FAO Statistics Data Base (www.faostat.org) (9 de noviembre de 2007).

Finlay K W, G N Wilkinson (1963) The analysis of adaptation in a plant breeding programme. Austr. J. Agric. Res. 14:742-754.

Lépiz I R, F Navarro J (1983) Frijol en el Noroeste de México (Tecnología de Producción). Ed. Impre-Jal. Guadalajara, Jalisco, México. 218 p.

Márquez S F (1974) El Problema de la Interacción Genético Ambiental en Genotecnia Vegetal. Ed. Patena, Escuela Nacional de Agricultura. Chapingo, México.

Padilla V I, I Armenta, J A Ramírez, M Madrid (2000) Tecnología para Producir Frijol en el Sur de Sonora. Folleto para Productores No 17. SAGARPA-INIFAP-Campo Experimental Valle del Mayo. Navojoa, Sonora, México. 24 p.

Rojo L J A (2002) Situación y perspectivas del frijol. In: Curso de Tecnología de Producción de Frijol. CEVACU-INIFAP- 
Fundación Produce Sinaloa. Culiacán. Sin. México. pp:4271.

SIAP (2005) Servicio de Información y Estadística Agroalimentaria y Pesquera. www.siea.sagarpa.gob.mx $(10$ de octubre de 2005).

Schwartz F H, G E Gálvez (1979) Problemas de Producción de Frijol: Enfermedades, Limitaciones Edáficas y Climáticas de Phaseolus vulgaris. Centro Internacional de Agricultura Tropical, Cali, Colombia. pp:211-298.

Valenzuela L J (1985) Parámetros de estabilidad para el rendimiento de variedades de frijol Phaseolus vulgaris L. en cuatro fechas de siembra. Agric. Téc. Méx. 11:185-200.
Valenzuela L J, I Armenta C (1990) Guía para Cultivar Frijol en el Sur de Sonora. Folleto para productores No 1. Campo Experimental Valle del Mayo. SAGARPA-INIFAP-CIANO. Cd. Obregón, Sonora, México. 16 p.

Voysest $O$ (1985) Frijol: Investigación y Producción. M López, F Fernández, A A Schoonhoven (eds). Centro de Internacional de Agricultura Tropical. Cali, Colombia. $418 \mathrm{p}$.

Welsh J R (1990) Fundamentals of Plant Genetics and Breeding. Colorado State University. Robert E. Krieger Publishing Company. Malabar, Florida, USA. 290 p. 